\title{
Valor predictivo de la hipocalcemia en el diagnóstico de la insuficiencia respiratoria aguda en COVID-19 en un hospital de segundo nivel de atención.
}

\author{
Predictive value of hypocalcemia in the diagnosis of acute respiratory failure in COVID-19 in a \\ second-level care hospital.
}

Tomás Noel Santana-Téllez',2, Milton Rojas-
García', Angie Yohana Del Águila-Grández',3.
Santana-Téllez T, Rojas-García M, Del Águila-Grández AY. Valor predictivo de la hipocalcemia en el diagnóstico de la insuficiencia respiratoria aguda en COVID-19 en un hospital de segundo nivel de atención. Rev Soc Peru Med Interna. 2021;34(4): 139-145. DOI: https://doi.org/10.36393/spmi.v34i4.629

\section{RESUMEN}

Objetivo. Demostrar la validez de la hipocalcemia para predecir la gravedad de la insuficiencia respiratoria en pacientes con COVID- 19. Materiales y métodos. Estudio tipo cohorte prospectivo, en 267 pacientes hospitalizados en la Unidad de Cuidados Intermedios del Hospital II EsSalud Pucallpa, entre enero y junio de 202I. Se extrajeron datos demográficos, clínicos y laboratoriales de las historias clínicas individuales. Se utilizó análisis multivariado para identificar las variables asociadas a la insuficiencia respiratoria aguda. Resultados. De los 267 pacientes, el 6I \% tuvo hipocalcemia al ingreso. En este grupo predominaron la tos seca, fatiga y dolor torácico; la fiebre y la disnea en la totalidad de los pacientes. La principal comorbilidad fue la obesidad. Tanto la frecuencia respiratoria $\geq 30 \mathrm{rpm}$ como la $\mathrm{SaO}_{2} \leq 93$ \% fueron más frecuentes en este grupo. Hubo relación inversa entre la hipocalcemia y la elevación de ciertos parámetros considerados marcadores inflamatorios; además de una mayor tasa de $\mathrm{PaFiO}_{2}$ anormal. Se constató una buena concordancia entre la hipocalcemia y los resultados de la $\mathrm{PaFiO}_{2}$ (índice Kappa de Cohen $=$ $0,63)$. Conclusiones. La hipocalcemia puede considerarse un factor predictor de insuficiencia respiratoria grave en pacientes con COVID-I9.

Palabras clave: Hipocalcemia, COVID-19, insuficiencia respiratoria aguda.

\section{ABSTRACT}

Objective. To demonstrate the validity of hypocalcemia to predict the severity of respiratory failure in patients with COVID-19. Materials and methods. Prospective cohort-type study in 267 patients hospitalized in the Intermediate Care Unit of Hospital II EsSalud Pucallpa, between January and June 202I. Demographic, clinical and laboratory data were extracted from individual medical records. Multivariate analysis was used to identify variables associated with acute

Médico internista. Hospital II EsSalud Pucallpa. Perú. Facultad de

Medicina Humana de la Universidad Nacional de Ucayali. Perú.

Orcid: https://orcid.org/0000-0002-8893-5489

2 Médico de medicina general integral y cardiólogo. Hospital II EsSalud

Pucallpa. Perú.

Orcid: https://orcid.org/0000-0002-5442-8286

3 Médica anestesióloga. Hospital II EsSalud Pucallpa. Perú.

Orcid: https://orcid.org/0000-0002-3939-9034 
respiratory failure. Results. Of the 267 patients, $61 \%$ had hypocalcemia on admission. In this group, dry cough, fatigue and chest pain predominated; fever and dyspnea in all patients. The main comorbidity was obesity. Both respiratory rate $\geq$ $30 \mathrm{bpm}$ and $\mathrm{SaO}_{2} \leq 93 \%$ were more frequent in this group. There was an inverse relationship between hypocalcemia and the elevation of certain parameters considered inflammatory markers; in addition to a higher rate of abnormal $\mathrm{PaFiO}_{2}$. There was a good concordance between hypocalcemia and $\mathrm{PaFiO}_{2}$ results (Cohen's Kappa index $=0.63$ ). Conclusions. Hypocalcemia can be considered a predictor of severe respiratory failure in patients with COVID-19.

Key words: Hypocalcemia, $2019 \mathrm{nCoV}$ disease, acute respiratory insufficiency

\section{INTRODUCCIÓN}

En el inicio del año 2020, el mundo se conmocionó con el reporte del fallecimiento, en Wuhan, China, de 27 pacientes a causa de una neumonía atípica, altamente agresiva, que no solo cambió notablemente la vida de los habitantes de todo el mundo sino que se convirtió en uno de los más grandes problemas sanitarios de la historia. Esta enfermedad, denominada COVID-19, es producida por un betacoronavirus (SARS-Cv-2), ya ha afectado a más de 200 millones de personas en todo el mundo, de los cuales, al menos, cuatro millones han fallecido. ${ }^{1,2}$

La COVID-19, es causada por el coronavirus del síndrome respiratorio agudo severo, cuya constelación clínica incluye fiebre, tos, astenia, artro-mialgia. Aproximadamente, el $80 \%$ de los pacientes cursa con una enfermedad leve o asintomática, con pronóstico favorable. Sin embargo; la prevalencia de insuficiencia respiratoria hipóxica en COVID-19 se ha presentado hasta en un $19 \%$, llegando a ventilación mecánica entre un 2,3-12,0\%. ${ }^{3}$

La mayoría de los conocimientos actuales, datan de los primeros planteamientos de un grupo de investigación liderado por Gattinoni, en cuyos trabajos describieron la presentación de la COVID-19 como un síndrome de dificultad respiratoria "atípico", con hipoxemia secundaria al estado de hiperperfusión/shunt, un parámetro que alcanza cifras de $3 \pm 2,1$ evidenciando tejido no aireado. Con este estudio, se clasifican los pacientes en dos espectros de expresión: el grupo de pacientes, denominados L, con mejor respuesta a los incrementos de la $\mathrm{FiO}_{2}$ y el grupo $\mathrm{H}$, con una mayor progresión y letalidad., ${ }^{4,5}$ Múltiples estudios han intentado caracterizar esta enfermedad; sin embargo, los elementos más investigados continúan siendo el papel que juegan los marcadores inflamatorios conocidos en su patogenia y su asociación con la progresión y la mortalidad. ${ }^{6}$ El calcio es uno de los factores implicados en los mecanismos de infección y replicación viral. Entre los mecanismos implicados está la interacción del calcio con los ácidos grasos insaturados, que son liberados como parte del proceso inflamatorio y que no se relaciona con déficit crónico de vitamina $\mathrm{D}$ o de hipoparatiroidismo.?

En un estudio realizado por Booth et al se constató hipocalcemia hasta en el $60 \%$ de los pacientes que ingresaron al hospital y cuya cifra ascendió hasta el $70 \%$ durante su período de hospitalización, ${ }^{8}$ lo cual coincide con otros estudios realizados en Europa y Estados Unidos. La hipocalcemia y su relación con la gravedad en enfermos críticos es un hecho comprobado, lo que ha servido de base para evaluar esta relación en pacientes con COVID-19; sin embargo, falta mucho por precisar y estudios por realizar. ${ }^{9,10}$ Esta investigación reporta los resultados obtenidos de un análisis de pacientes hospitalizados con diagnóstico confirmado de neumonía COVID-19 cuyo objetivo fue demostrar la validez de la hipocalcemia para predecir la gravedad de la insuficiencia respiratoria por COVID-19.

\section{MATERIAL Y MÉTODOS}

Tipo de estudio

Se estudió una cohorte, prospectiva, con análisis transversal, que se ejecutó en la Unidad de Cuidados Intermedios del Hospital II EsSalud Pucallpa en el período comprendido entre el 1 de enero al 30 de junio de 2021.

Población y muestra

La muestra quedó constituida por los 267 pacientes que cumplieron con los criterios de inclusión definidos para la presente investigación. En el cálculo muestral se tuvo en cuenta que se trabajó con una población definida ( $\mathrm{N}=874)$, utilizándose la fórmula correspondiente para este caso.

$\mathrm{n}=\mathrm{NZ} \mathrm{Z}^{2} \mathrm{PQ} /\left[\mathrm{d}^{2}(\mathrm{~N}-1)+\mathrm{Z}^{2} \mathrm{PQ}\right]$

Criterios de inclusión

Se incluyeron todos los pacientes con el diagnóstico de COVID-19 hospitalizados en la Unidad de Cuidados Intermedios del hospital, en el período antes mencionado, a quienes se les determinó el calcio ionizado en la hemogasometría arterial.

Criterios de exclusión

- Pacientes a los cuales no se les determinó niveles de calcemia.

- Pacientes cuya historia clínica no contenía las variables a estudiar.

- Pacientes con alguna enfermedad que se asocie a trastorno del calcio.

Variables de estudio

Se consideró como variable dependiente la insuficiencia respiratoria aguda durante la hospitalización $\left(\mathrm{PaFiO}_{2}>300\right.$ mmHg - normal, 201-299 mmHg - insuficiencia respiratoria ligera, 101-199 $\mathrm{mmHg}$ - insuficiencia respiratoria moderada, $\leq 100 \mathrm{mmHg}$ - insuficiencia respiratoria severa). La variable independiente fue la hipocalcemia, medida a través de niveles de calcio ionizado reales como los ajustados a un $\mathrm{pH}$ de 7,4 y las variables intervinientes fueron la edad (en años), sexo (masculino/femenino), 
comorbilidades (respuesta dicotómica: sí/no; obesidad, hipertensión arterial, enfermedades cardiovasculares, diabetes mellitus tipo 2, neumopatía crónica), saturación de oxígeno $\left(\mathrm{SaO}_{2} ; \geq 93 \% /<93 \%\right)$, manifestaciones clínicas (respuesta dicotómica: si/no; fiebre, tos, expectoración, fatiga, disnea, dolor torácico, cefalea, diarrea), hallazgos de laboratorio (respuesta dicotómica: normal/anormal según valores normales estandarizados de la institución; ferritinemia, fibrinogenemia, lactato deshidrogenasa LDH, transaminasas (TGP, TGO), proteína $\mathrm{C}$ reactiva (PCR), recuento absoluto de linfocitos (RAL), creatinina, conteo de plaquetas).

Fuentes de datos/medidas

Se tuvieron en cuenta tanto los niveles de calcio ionizado reales como los ajustados a un $\mathrm{pH}$ de 7,4 , con el objetivo de evitar la influencia de la posible manipulación de la muestra.

$\mathrm{CapH} \_\mathrm{c}=\mathrm{Ca}^{2+}+[0,12 \times(\mathrm{pH}-7,4) / 0,1]$

La hipocalcemia se definió como un nivel de calcio por debajo de $1,18 \mathrm{mmol} / \mathrm{L}$.
Descripción de los procedimientos e instrumentos para la recolección de datos

Se diseñó un cuestionario, con las variables del estudio, cuyo llenado se realizó a partir de la búsqueda en el registro general de pacientes hospitalizados desde el 1 de enero al 30 de junio de 2021, en el área antes mencionada. Una vez identificados, se revisaron sus historias clínicas electrónicas a través del programa Servicio de salud inteligente, que permite revisar las evoluciones, los exámenes auxiliares, diagnósticos, prescripciones y tratamientos administrados. Tres investigadores realizaron el registro de los datos; de ellos, uno, realizó todo el análisis estadístico del estudio.

Aspectos éticos

Se aseguró la confidencialidad de la información obtenida a través de la aplicación del cuestionario creado al efecto, respetando los principios éticos normados en la declaración de Helsinki, para la investigación en humanos. Se solicitó la correspondiente autorización de la Oficina de Capacitación y Docencia de la institución. No se solicitó el consentimiento informado, debido al tipo de estudio

Tabla I. Características sociodemográficas y clínicas de los pacientes estudiados.

\begin{tabular}{|c|c|c|c|c|}
\hline Variables & $\begin{array}{l}\text { Hipocalcen } \\
\text { Si }(n=163)\end{array}$ & No $(n=104)$ & OR (IC $95 \%)$ & Valor $\mathbf{p}$ \\
\hline Edad (media en años) & $62( \pm 6,3)$ & $52( \pm 3,8)$ & NA & 0,00 \\
\hline Calcemia (mmol/L) & 1,03 & 1,8 & NA & 0,00 \\
\hline \multicolumn{5}{|l|}{ Sexo } \\
\hline Masculino & 124 & 69 & $5,6(3,2-9,8)$ & 0,00 \\
\hline Femenino & 59 & 35 & & \\
\hline \multicolumn{5}{|l|}{ Comorbilidades } \\
\hline Obesidad & $74(45,4 \%)$ & $38(36,5 \%)$ & $1,4(0,9-2,4)$ & 0,15 \\
\hline Hipertensión arterial & $49(30,1 \%)$ & $17(16,3 \%)$ & $2,2(1,2-4,1)$ & 0,01 \\
\hline Diabetes mellitus & II $(6,7 \%)$ & $3(2,9 \%)$ & $2,4(0,7-8,9)$ & 0,16 \\
\hline Enfermedad coronaria & $4(2,5 \%)$ & $2(1,9 \%)$ & I,3 (0,2-7, I & 0,77 \\
\hline Neumopatía crónica & $2(1,2 \%)$ & I (I \%) & $I, 3(0, I-14,3)$ & 0,84 \\
\hline \multicolumn{5}{|l|}{ Síntomas y signos } \\
\hline Fiebre & $163(100 \%)$ & 104 (100\%) & & \\
\hline Tos & 147 (90,2%) & $68(65,4 \%)$ & $4,9(2,5-9,4)$ & 0,00 \\
\hline Expectoración & $49(30,1 \%)$ & $23(22,1 \%)$ & I,5 $(0,9-2,74)$ & 0,15 \\
\hline Fatiga & 159 (97,5 \%) & 78 (75 \%) & $13,3(4,5-39,3)$ & 0,00 \\
\hline Disnea & $163(100 \%)$ & 104 (100\%) & & \\
\hline Dolor torácico & 120 (73,6 \%) & $43(41,3 \%)$ & $3,9(2,3-6,7)$ & 0,00 \\
\hline Cefalea & 31 (19\%) & $19(18,3 \%)$ & $\mathrm{I}, \mathrm{I}(0,6-2)$ & 0,87 \\
\hline Diarrea & $27(16,6 \%)$ & 27 (26 \%) & $0,6(0,3-1)$ & 0,06 \\
\hline \multicolumn{5}{|l|}{ Frecuencia respiratoria } \\
\hline$\geq 30 \mathrm{rpm}$ & $95(59,3 \%)$ & $28(26,9 \%)$ & $3,8(2,2-6,5)$ & 0,00 \\
\hline$<30 \mathrm{rpm}$ & 68 (4I,7 \%) & $76(74,1 \%)$ & & \\
\hline \multicolumn{5}{|l|}{$\mathrm{SaO}_{2}$} \\
\hline$\leq 93 \%$ & II 8 (72,4%) & $53(50,9 \%)$ & $2,5(1,5-4,2)$ & 0,00 \\
\hline$>93 \%$ & $45(27,6 \%)$ & 5 I (49,1 \%) & & \\
\hline
\end{tabular}

Frecuencia cardíaca

Fuente: Registro primario. 
(observacional - retrospectivo).

Métodos estadísticos

El análisis estadístico de los datos se realizó utilizando el programa SPSS (Statistical Package for Social Sciences) versión 26. Para el análisis descriptivo, se calculó tanto la media y la desviación estándar para las variables cuantitativas como las frecuencias absolutas y relativas para las variables cualitativas. Se determinó la asociación entre las variables cualitativas mediante el uso de la prueba chi ${ }^{2}$ de Pearson, la prueba exacta de Fisher, en función de las condiciones de aplicación. Para determinar la concordancia entre la hipocalcemia y la insuficiencia respiratoria aguda se utilizó el test de Kappa de Cohen. Se trabajó con un 95 $\%$ de confiabilidad y un nivel de significancia estadística $(\mathrm{p} \leq 0,05)$.

\section{RESULTADOS}

En el presente estudio se incluyeron 267 pacientes con diagnóstico confirmado de COVID-19, de los cuales 163
(61\%) tuvieron hipocalcemia al ingreso, con una calcemia media de 1,03 mmol/L. La tabla 1 muestra las principales características sociodemográficas y clínicas. En el grupo con hipocalcemia hubo predominio de la tos seca, fatiga y dolor torácico; estando presente la fiebre y la disnea en la totalidad de los pacientes (incluso de ambos grupos). En ambos grupos, la obesidad fue la principal comorbilidad asociada; sin que hubiera diferencias significativas entre ellos, siendo la hipertensión arterial predominante en pacientes con hipocalcemia. La polipnea (frecuencia respiratoria $\geq 30 \mathrm{rpm}$ ) y la $\mathrm{SaO} 2 \leq 93 \%$ fueron más frecuentes en este grupo.

En la evaluación laboratorial al ingreso se pudo constatar una relación inversa entre la hipocalcemia y la elevación de ciertos parámetros considerados marcadores inflamatorios; entre ellos, la ferritina, fibrinógeno, $\mathrm{LDH}$, transaminasas y $\operatorname{PCR}(p=0,00)$. En relación con el recuento absoluto de linfocitos, en ambos grupos, hubo linfopenia, siendo significativamente más bajos en pacientes con hipocalcemia.

Tabla 2. Hallazgos de laboratorio en los pacientes estudiados.

\begin{tabular}{|c|c|c|c|c|}
\hline Variables & $\begin{array}{l}\text { Hipocalcemia } \\
\text { Si }(n=163)\end{array}$ & No $(n=104)$ & OR (IC $95 \%$ ) & Valor $\mathbf{p}$ \\
\hline \multicolumn{5}{|l|}{ Ferritinemia } \\
\hline Elevada & 94 & 45 & I,8 (I,I-2,9) & 0,02 \\
\hline Normal & 69 & 59 & & \\
\hline \multicolumn{5}{|l|}{ Fibrinogenemia } \\
\hline Elevada & 134 & 47 & $5,6(3,2-9,8)$ & 0,00 \\
\hline Normal & 29 & 57 & & \\
\hline \multicolumn{5}{|l|}{ LDH } \\
\hline Elevada & 154 & 65 & $10,2(4,7-22,4)$ & 0,00 \\
\hline Normal & 9 & 39 & & \\
\hline \multicolumn{5}{|l|}{ TGP } \\
\hline Elevada & 108 & 53 & I,9 (I,I-3,I) & 0,01 \\
\hline Normal & 55 & 51 & & \\
\hline \multicolumn{5}{|l|}{ TGO } \\
\hline Elevada & 100 & 43 & $2,3(1,4-3,7)$ & 0,00 \\
\hline Normal & 63 & 61 & & \\
\hline \multicolumn{5}{|l|}{ PCR } \\
\hline Elevada & 158 & 82 & $6, I(2,5-14,8)$ & 0,00 \\
\hline Normal & 7 & 22 & & \\
\hline \multicolumn{5}{|l|}{ RAL } \\
\hline Normal & 135 & 64 & $3(1,7-5,3)$ & 0,00 \\
\hline Linfopenia & 28 & 40 & & \\
\hline \multicolumn{5}{|l|}{ Creatinina } \\
\hline Elevada & 88 & 61 & $0,8(0,5-I, 3)$ & 0,36 \\
\hline Normal & 78 & 43 & & \\
\hline \multicolumn{5}{|l|}{ Conteo de plaquetas } \\
\hline Trombocitopenia & 56 & 44 & $0,7(0,4-I, 2)$ & 0,19 \\
\hline Normal & 107 & 60 & & \\
\hline
\end{tabular}

Fuente: Registro primario. 
Tabla 3. Relación entre calcemia y $\mathrm{PaFiO}_{2}$.

\begin{tabular}{lllll} 
PaFio $_{2}$ & Hipocalcemia & Total & Valor p \\
Normal & Si & No & & \\
\hline Insuficiencia leve & $13(4,8 \%)$ & $45(16,8 \%)$ & $58(21,6 \%)$ & \\
\hline Insuficiencia moderada & $25(9,4 \%)$ & $24(9 \%)$ & $49(18,4 \%$ & \\
Insuficiencia severa & $76(18,4 \%)$ & $21(7,9 \%)$ & $70(26,3 \%)$ & \\
Total & $163(61,1 \%)$ & $104(39,9 \%)$ & $267(100 \%)$ & 0,00 \\
Fuente: Registro primario. & & & &
\end{tabular}

Tabla 4. Validez de la hipocalcemia como predictor de insuficiencia respiratoria aguda en COVID-19.

\begin{tabular}{|c|c|c|c|}
\hline Hipocalcemia & $\begin{array}{l}\mathrm{PaFiO}_{2} \\
\text { Baja }(<300) *\end{array}$ & Normal ( $\geq 300) *$ & Total \\
\hline $\mathrm{Si}$ & 150 & 13 & 163 \\
\hline No & 45 & 59 & 104 \\
\hline \multirow[t]{2}{*}{ Total } & 195 & 72 & 267 \\
\hline & $\%$ & & \\
\hline Prevalencia & 68,2 & & \\
\hline Sensibilidad & 82,4 & & \\
\hline Especificidad & 84,7 & & \\
\hline Valor predictivo positivo & 92 & & \\
\hline Valor predictivo negativo & 69,2 & & \\
\hline Cociente de probabilidad positivo & 5,4 & & \\
\hline $\begin{array}{l}\text { Cociente de probabilidad negativo } \\
\text { p=0,00; índice de Kappa de Cohen=0,63. } \\
\text { * mm Hg. }\end{array}$ & 0,2 & & \\
\hline
\end{tabular}

Las alteraciones de la creatinina sérica y del conteo plaquetario no constituyeron hallazgos significativos en la presente investigación (tabla 2).

La tabla 3 muestra la relación entre la hipocalcemia y la $\mathrm{PaFiO}_{2}$, que evidencia el estado de la función respiratoria y por ende de la gravedad de la misma. Nótese, que en el grupo de pacientes con hipocalcemia hubo una mayor progresión hacia la insuficiencia respiratoria aguda severa (28,5\%) que, en el otro grupo, donde se encontraron más pacientes con $\mathrm{PaFiO}_{2}$ normal (16,8\%).

La tabla 4 expresa la concordancia entre la hipocalcemia y los resultados de la $\mathrm{PaFiO} 2$; se observa una buena concordancia entre ambas pruebas para el diagnóstico de insuficiencia respiratoria aguda en COVID-19 (índice Kappa de Cohen=0,63). La hipocalcemia evidenció una sensibilidad del $82,4 \%$ y una especificidad del $84,7 \%$ para predecir este diagnóstico (insuficiencia respiratoria aguda). Siendo el valor predictivo positivo (VPP) del $92 \%$, el valor predictivo negativo (VPN) del 69,2\%, el cociente de probabilidad positivo del 5,4\% y el cociente de probabilidad negativo del $0,2 \%$.

\section{DISCUSIÓN}

La COVID-19, desde inicio de 2020, se convirtió en una amenaza sanitaria de gran magnitud; hasta la fecha ha producido la muerte de 4627288 personas en todo el mundo, de los cuales el 31,4\% pertenece a América Latina. ${ }^{11}$

En varios estudios, se ha constatado la presencia de hipocalcemia en pacientes con COVID-19, tanto en emergencias como en servicios de hospitalización, llegando incluso a ser muy frecuente en los casos graves. ${ }^{12-14} \mathrm{La}$ elevación de los ácidos grasos insaturados (UFA, siglas en inglés) determina ciertos efectos proinflamatorios e inducción de la llamada tormenta citoquínica, la cual se ha relacionado, a través de mecanismos no bien conocidos, con la gravedad de esta enfermedad. ${ }^{7}$ Di Filippo et al, al estudiar dos grupos de pacientes con las mismas características clínicas basales y parámetros inflamatorios, uno con COVID-19 y otro sin ella, observaron que el 40 $\%$ del grupo COVID-19 presentaba hipocalcemia, incluso con niveles más bajos de calcemia al compararlo con los no COVID-19, lo que conllevó a una peor dificultad respiratoria. ${ }^{10}$ 
En una investigación realizada por Chen et al, se constató una mayor prevalencia de ciertas manifestaciones clínicas, entre las que sobresalieron la disnea, la polipnea, la presencia de $\mathrm{SaO}_{2} \leq 93 \%$ y la $\mathrm{PaFiO}_{2}<300 \mathrm{~mm} \mathrm{Hg}$, con significancia estadística en los casos más severos $(\mathrm{p}<$ $0,05)$, hecho que coincide con resultados obtenidos por Richardson et al, quienes confirmaron estos hallazgos en pacientes hospitalizados con COVID-19. ${ }^{15,16}$

Plasencia et al, luego de realizar una revisión sistemática, que incluyó 13 estudios con 99817 pacientes, comprobaron la asociación de la COVID-19 con varias comorbilidades; entre ellas, la hipertensión arterial como la principal (RP 4,05; IC $95 \%$ ), seguida de la enfermedad cardiovascular, la diabetes mellitus y el hábito de fumar, respectivamente. ${ }^{7}$ La posible participación del eje renina-angiotensina, en particular la utilización de la enzima convertidora de angiotensina $2\left(\mathrm{ACE}_{2}\right.$, siglas en inglés) como receptor del SARS-CoV-2 y su alta expresión explicarían la asociación con otras comorbilidades, entre ellas la hipertensión arterial, la enfermedad renal crónica, la enfermedad cardiovascular y la diabetes mellitus. De igual manera y de modo significativo, esta asociación ha sido reportada para otras enfermedades respiratorias, lo que tiene relevancia patogénica en la COVID-19. ${ }^{18-20}$

Según Mejía et al, en un estudio realizado en el Hospital Cayetano Heredia, de Lima, el 68,6 \% de su muestra tuvo al menos una comorbilidad, principalmente la obesidad, la diabetes mellitus e hipertensión arterial, y observaron que la hipoxemia se comporta como un factor independiente de mortalidad. ${ }^{21}$ Entre los exámenes auxiliares, se evidenció leucocitosis con linfopenia relativa asociada e incremento de marcadores inflamatorios, entre ellos, la proteína $\mathrm{C}$ reactiva y la deshidrogenasa láctica. La mayoría de los pacientes requirieron soporte oxigenatorio de alto flujo $\left(\mathrm{FiO}_{2} \geq 0,36\right)$. Hueda-zavaleta et $\mathrm{al}$, en un estudio en hospitalizados con diagnóstico confirmado de neumonía por SARS-CoV-2, también constataron la asociación de la COVID-19 con ciertas comorbilidades, principalmente la obesidad, hipertensión y diabetes. ${ }^{22}$ Además, los factores de riesgo de mortalidad fueron la $\mathrm{SaO}_{2}<90 \%$ y el aumento de la deshidrogenasa láctica.

Liu et al comprobaron que la hipocalcemia fue más común en pacientes con características clínicas y de laboratorio de COVID-19 severa, además de peores resultados pronósticos. ${ }^{23}$ En ese estudio la hipocalcemia ocurrió en el $62,6 \%$ de los pacientes, sin que hubiera diferencia significativa en relación con la edad, manifestaciones clínicas y comorbilidades al compararse con los pacientes normocalcémicos. En relación con los marcadores de laboratorio, se observó una significativa diferencia estadística entre ambos grupos en el conteo de leucocitos, la elevación de PCR, de la procalcitonina, del dímero D, así como la hipoalbuminemia.

Singh et al, en relación con el artículo de Liu et al, reforzaron el papel de la hipocalcemia, la hipoalbuminemia y la tormenta de citocinas en la severidad de la insuficiencia respiratoria por COVID-19, de manera que la suplementación precoz de calcio y albúmina, con el objetivo de mantener sus niveles normales, pudiera prevenir la progresión a la infección grave y que si esta acción se lleva a cabo fuera de este período no tendría ningún valor. ${ }^{24}$ Torres et al reportaron la alta frecuencia de hipocalcemia en los pacientes hospitalizados con COVID-19 (63\%) y que permite la predicción de un peor pronóstico si ella se constata en las primeras 72 horas del ingreso. ${ }^{25}$ Además, el grupo con hipocalcemia requirió alto soporte de oxígeno durante la hospitalización (49\%) al compararlo con el grupo normocalcémico (32\%) y tuvieron más ingresos en UCI ( $42 \%$ vs $26 \%$; $p=0,005)$, sin que hubiera diferencias en la mortalidad entre los grupos.

En el estudio de Cutillas et al, el 61,6\% de los pacientes incluidos tuvo un calcio iónico bajo, de ellos el $1 \%$ con hipocalcemia grave y el 60,6 \% moderada, y con asociación entre la prevalencia del síndrome de dificultad respiratoria aguda (SDRA) y del ingreso a la unidad de cuidados intensivos (UCI) en los pacientes con hipocalcemia al ser comparado con aquellos que presentaron normocalcemia. ${ }^{26}$ Además, se evidenció correlación negativa entre los niveles de calcio iónico y los de LDH, ferritina, IL-6 y PCR.

No se ha reportado estudio alguno que haya definido la validez de la hipocalcemia como factor predictor de gravedad de la COVID-19. En el presente estudio observamos una evidente asociación entre la hipocalcemia y ciertas comorbilidades, como la obesidad y la hipertensión arterial, además de una relación inversa con la elevación de algunos marcadores considerados como proinflamatorios. En particular, se evidenció a través del índice de concordancia de Kappa de Cohen que la presencia de hipocalcemia al ingreso o en las primeras 72 horas de hospitalización tiene un valor predictivo elevado para insuficiencia respiratoria severa, lo cual permitirá tenerlo como un marcador independiente para evitar la progresión a formas graves de esta enfermedad.

En conclusión, la hipocalcemia puede considerarse como un factor predictor de insuficiencia respiratoria aguda grave en pacientes con neumonía COVID-19.

\section{REFERENCIAS BIBLIOGRÁFICAS}

I. Zhu N, Zhang D,Wang W, et al.A Novel Coronavirus from Patients with Pneumonia in China, 2019. N Engl J Med. 2020;382(8):727-733. doi: I0.1056/NEJMoa200I0I7.

2. Gralinski LE, Menachery VD. Return of the coronavirus: 2019-nCoV. Viruses. 2020; I2(2):EI 35.

3. Zhou P,Yang XL,Wang XG. et al. A pneumonia outbreak associated with a new coronavirus of probable bat origin. Nature. 2020;579, 270-273. https://doi.org/I0.1038/s4I586-020-20I2-7.

4. Gattinoni L, Chiumello D, Caironi P, Busana M, Romitti F, Brazzi L, et al. COVID-19 pneumonia: different respiratory treatments for different phenotypes? Intensive Care Med. 2020;46(6): I099-I02.

5. Gattinoni L, Coppola S, Cressoni M, Busana M, Rossi S, Chiumello D. COVID-19 Does not lead to a «typical» acute respiratory distress syndrome. Am J Respir Crit Care Med. 2020;20I(I0):I299. 300.

6. Di Filippo L, Formenti AM, Rovere-Querini P, et al. Hypocalcemia is highly prevalent and predicts hospitalization in patients with COVID-I9. Endocrine. 2020;68(3):475-478. doi: I0. I007/s I 2020-02002383-5

7. Singh VP, Khatua B, El-Kurdi B, Rood C. Mechanistic basis and 
therapeutic relevance of hypocalcemia during severe COVID-19 infection. Endocrine. 2020;70(3):46I-462. doi:I0.1007/s I 2020-02002530-y

8. Booth CM, Matukas LM, Tomlinson GA, et al. Clinical features and short-term outcomes of I44 patients with SARS in the Greater Toronto area. JAMA. 2003;289(2I):280I-2809. doi: I0.100I/ jama.289.21.JOC30885.

9. Cheungpasitporn W,Thongprayoon C, Mao MA, Kittanamongkolchai W, Sakhuja A, Erickson SB. Impact of admission serum calcium levels on mortality in hospitalized patients. Endocr Res. 20 I8 May;43(2): I I 6I23. doi: I0.1080/07435800.20 I8.|433200. Epub 2018 Jan 30. PMID: 29381079.

10. di Filippo L, Formenti AM, Doga M, Frara S, Rovere-Querini P, Bosi $E$, et al. Hypocalcemia is a distinctive biochemical feature of hospitalized COVID-19 patients. Endocrine. 202I;7I(I):9-I3. doi: |0.|007/s | 2020-020-0254|-9.

II. Estadísticas del coronavirus en el mundo https://news.google.com/ covid I 9/map?hl=es-4 I 9\&gl=PE\&ceid=PE\%3Aes-4I 9

12. Filippo, Luigi \& Formenti, Anna \& Giustina, Andrea. (2020). Hypocalcemia: the quest for the cause of a major biochemical feature of COVID-19. Endocrine. 70. 463-464. 10.1007/s I 2020-02002525-9.

13. Bossoni S, Chiesa L, Giustina A. Severe hypocalcemia in a thyroidectomized woman with COVID-19 infection. Endocrine. 2020;68(2):253-254. doi: I0.1007/s I 2020-02002326-0.

14. Cappellini F, Brivio R, Casati M, Cavallero A, Contro E, Brambilla P. Low levels of total and ionized calcium in blood of COVID-19 patients. Clin. Chem. Lab. Med. 2020;58(9):el7I-I73. doi: 10.15I5/ cclm-2020-06I I.

15. Chen G, Wu D, Guo W, Cao Y, Huang D, Wang H, Wang T, Zhang X, Chen H, Yu H, Zhang X, Zhang M, Wu S, Song J, Chen T, Han M, Li S, Luo X, Zhao J, Ning Q. Clinical and immunological features of severe and moderate coronavirus disease 2019. J Clin Invest. 2020 May I; I30(5):2620-2629. doi: I0.I I72/JCII 37244. PMID: 322 I7835; PMCID: PMC7I 90990.

16. Richardson S, Hirsch JS, Narasimhan M, Crawford J, McGinn T, Davidson KW et al. Presenting characteristics, comorbidities, and outcomes among 5700 patients hospitalized with COVID-19 in the New York City Area. JAMA. 2020;323(20):2052-2059. doi:I0.100I/jama.2020.6775.

17. Plasencia-Urizarri TM, Aguilera-Rodríguez R, Almaguer-Mederos LE. Comorbilidades y gravedad clínica de la COVID-19: revisión sistemática y meta-análisis. Rev haban cienc méd [Internet]. 2020; 19(Suppl I): e3389. URL disponible en: http://scielo.sld.cu/ scielo.php?script=sci_arttext\&pid=S I 729-5 I 9X2020000400 $002 \&$ Ing=es. Epub 10-Jun-2020

18. Hoffmann M, Kleine Weber H, Schroeder S, Kruger N, Herrler T, Erichsen S, et al. SARS-CoV-2 cell entry depends on ACE2 and TMPRSS2 and is blocked by a clinically proven protease inhibitor. Cell. 2020;181:1-10.

19. Zhou P, Yang XL, Wang XG, Hu B, Zhang L, Zhang W, et al. A pneumonia outbreak associated with a new coronavirus of probable bat origin. Nature. 2020;579: 270-3.

20. Rao S, Lau A, So HC. Exploring diseases/traits and blood proteins causally related to expression ofACE2, the putative receptor of 2019nCov: A Mendelian randomization analysis [Internet]. MedRxiv. New York: Cold Spring Harbor; 2020. URL disponible en: https://2020.2003.2004. 2003 I 237

21. Mejía F, Medina C, Cornejo E, Morello E, Vásquez S, Alave J, et al. Oxygen saturation as a predictor of mortality in hospitalized adult patients with COVID-19 in a public hospital in Lima, Peru. PLoS One.2020; I 5(I 2):e0244 I 7I.doi: I0. I37 I/journal.pone.0244 I 7 I. » https://doi.org/10.1371/journal.pone.0244I7I

22. Hueda-Zavaleta M, Copaja-Corzo C, Bardales-Silva F, Flores-Palacios R, Barreto-Rocchetti L, Benites-Zapata VA. Factores asociados a la muerte por COVID-I9 en pacientes admitidos en un hospital público en Tacna, Perú. Rev perú med exp salud publica [Internet]. 202I;38(2): 2I4-223. URL disponible en: http://www.scielo.org.pe/ scielo.php?script=sci_arttext\&pid=S I 726-4634202 I 0002002 | 4 \&lng=es

23. Liu J, Han P,Wu J, Gong J, Tian D. Prevalence and predictive value of hypocalcemia in severe COVID-I9 patients. J Infect Public Health. 2020 Sep;I3(9):I224-1228. doi: 10.1016/j.jiph.2020.05.029. Epub 2020 Jun 22. PMID: 32622796 ; PMCID: PMC7306733.

24. Singh VP, Khatua B, El-Kurdi B. Hypocalcemia and hypoalbuminemia during COVID-19 infection: Opportunities for therapeutic intervention. J Infect Public Health. 2020; I 3(I2): I887. doi: I0.1016/j. jiph.2020.09.019. Epub 2020 Oct I0. PMID: 3328964I; PMCID: PMC7547564.klvjk.

25. Torres B, Alcubilla P, González-Cordón A, Inciarte A, Chumbita M, Cardozo C, et al. COVIDI9 Hospital Clínic Infectious Diseases Research Group. Impact of low serum calcium at hospital admission on SARS-CoV-2 infection outcome. Int J Infect Dis. 2021;104: I64168. doi: 10.1016/j.ijid.2020.II.207. Epub 2020 Dec 2. PMID: 33278624; PMCID: PMC7709580.

26. Cutillas Pérez MD, Sánchez Martínez JF, Pinar Sánchez J, Olague Baños C, García Parra G, Sánchez A. Prevalencia y significación clínica de la hipocalcemia y el déficit de vitamina $d$ en pacientes con COVID-19 en un hospital de segundo nivel. Rev Clin Esp. 202I;22I (Espec Congr):33.

\section{CORRESPONDENCIA:}

Tomás Noel Santana Tellez

tstcuba2013@yahoo.es

Fecha de recepción: 27-10-2021.

Fecha de aceptación: 22-I|-202I.

Declaración de conflicto de intereses: Los autores declaran que no existe conflicto de intereses.

Fuente de financiamiento:Autofinanciado. 